\title{
Triple Laplace Transform in Bicomplex Space with Application
}

\author{
Mahesh Puri Goswami ${ }^{1, *}$, Naveen Jha ${ }^{2}$ \\ ${ }^{1}$ Department of Mathematics \& Statistics, Mohanlal Sukhadia University, Udaipur-313001, India \\ ${ }^{2}$ Department of Mathematics, Government Engineering College, Bharatpur-321303, India
}

Received April 7, 2020; Revised June 2, 2020; Accepted June 16, 2020

Copyright $@ 2020$ by authors, all rights reserved. Authors agree that this article remains permanently open access under the terms of the Creative Commons Attribution License 4.0 International License

\begin{abstract}
In this article, we investigate bicomplex triple Laplace transform in the framework of bicomplexified frequency domain with Region of Convergence (ROC), which is generalization of complex triple Laplace transform. Bicomplex numbers are pairs of complex numbers with commutative ring with unity and zero-divisors, which describe physical interpretation in four dimensional space and provide large class of frequency domain. Also, we derive some basic properties and inversion theorem of triple Laplace transform in bicomplex space. In this technique, we use idempotent representation methodology of bicomplex numbers, which play vital role in proving our results. Consequently, the obtained results can be highly applicable in the fields of Quantum Mechanics, Signal Processing, Electric Circuit Theory, Control Engineering, and solving differential equations. Application of bicomplex triple Laplace transform has been discussed in finding the solution of third-order partial differential equation of bicomplex-valued function.
\end{abstract}

Keywords Bicomplex functions, Triple Laplace transform and Bicomplex Laplace transform.

2010 Mathematics Subject Classification Primary 30G35; Secondary 42B10.

\section{Introduction}

Enormous efforts have been done in past few years in the applications of bicomplex functions and fine research has been developed. The concept of bicomplex numbers was introduced by Segre [6], in order to compactly describe physical interpretation in four-dimensional space. Set of bicomplex numbers is a commutative ring with unity and zero divisors which contains the commutative ring of hyperbolic numbers and the field of complex numbers. In fact, bicomplex numbers are generalization of complex numbers and hyperbolic numbers [9]. Hence, adverse to quaternions, bicomplex numbers are commutative with some non-invertible elements interpolated on the null cone.

The concept of holomorphic functions of a bicomplex variable is generated by Futagawa $[15,16]$. Some fundamental results in the theory of bicomplex holomorphic functions were given by Dragoni [11] while Price [10] and Rönn [29] have evolved the bicomplex function theory and algebra.

Recently, enormous efforts have been done to expand the theory of integral transforms in bicomplex space and studied their applications by Agarwal et al. [19, 20, 21, 23]. However, in recent bicomplex Schödinger equation and some of its properties studied by Rochon and Trembley [8] and self adjoint operators were defined for finite and infinite dimensional bicomplex Hilbert spaces [9, 26, 27]. An analytical method to solve bicomplex version of Schrödinger equation corresponds to the Hamiltonian system was studied by Banerjee [2]. Lavoie et al. [28] examined the quantum harmonic oscillator problem in bicomplex numbers and obtained eigenvalues and eigenkets of the bicomplex harmonic oscillator. Kumar et al. [25] introduced the bicomplex version of topological vector spaces and topological modules were developed by Kumar and Saini [24] over the ring of bicomplex numbers. Cerejeiras et al. [17] reconstructed a bicomplex sparse signal with high probability from a reduced number of bicomplex random samples. Ghanmi and Zine [4] introduced bicomplex Segal-Bargmann and fractional Fourier transforms.

Double Laplace transform proposed by Van der Pol [31] and applied by Humbert [18] in the study of hypergeometric functions; by Jaeger [12] to solve boundary value problems in heat conduction. The complex double Laplace transform was expanded to multiple Laplace transform in $n$ independent complex variables by Estrin and Higgins [30]. Applications of triple Laplace transform in solving third order partial differential Mboctara equation was discussed by Atangana [1]. Agar- 
wal et al. [22] generalized double Laplace transform to bicomplex double Laplace transform and found some applications.

For solving the large class of partial differential equations of bicomplex-valued function, we require integral transforms defined for large class. In this procedure we derive triple Laplace transform in bicomplex space with ROC that can be competent the transferring signals from real-valued $(x, y, z)$ domain to bicomplexified frequency $(\xi, \eta, \gamma)$ domain.

This article is organised as follows:

In Section 3, we introduce triple Laplace transform in bicomplex space with ROC. Some fundamental properties of triple Laplace transform in bicomplex space are presented in Section 4. In Section 5, we introduce the inversion formula for triple Laplace transform in bicomplex space. In Section 6, we discuss application of bicomplex triple Laplace transform in finding the solution of third-order partial differential equations of bicomplex-valued functions and last Section 7 contains the conclusion.

\section{Preliminaries on Bicomplex Numbers}

We start with an unconventional interpretation of the set of complex numbers $\mathbb{C}$ which is ordered pair of two real numbers in complex plane with a non-real unit $i_{1}$ s.t. $i_{1}^{2}=-1$ as follows

$$
\mathbb{C}=\left\{z=x+i_{1} y: x, y \in \mathbb{R}\right\},
$$

where $\mathbb{R}$ is the set of real numbers. In similar way, the set of bicomplex numbers $\mathbb{C}_{2}$ which is ordered pair of two complex numbers with non-real units $i_{1}$ and $i_{2}$ s.t. $i_{1}^{2}=i_{2}^{2}=-1$, $i_{1} i_{2}=i_{2} i_{1}=j, j^{2}=1$ as follows

$$
\mathbb{C}_{2}=\left\{\xi=z_{1}+i_{2} z_{2}: z_{1}, z_{2} \in \mathbb{C}\right\} .
$$

Or

$$
\mathbb{C}_{2}=\left\{\xi=x_{0}+i_{1} x_{1}+i_{2} x_{2}+j x_{3}: x_{0}, x_{1}, x_{2}, x_{3} \in \mathbb{R}\right\} .
$$

An important characteristic of bicomplex numbers is the unambiguous representation using the idempotent elements $e_{1}=$ $\frac{1+i_{1} i_{2}}{2}$ and $e_{1}=\frac{1-i_{1} i_{2}}{2}$ with $e_{1}+e_{2}=1$ and $e_{1} e_{2}=e_{2} e_{1}=0$. In fact, for every $\xi \stackrel{2}{=} z_{1}+i_{2} z_{2} \in \mathbb{C}_{2}$, we get

$$
\begin{aligned}
z_{1}+i_{2} z_{2} & =\left(z_{1}-i_{1} z_{2}\right) e_{1}+\left(z_{1}+i_{1} z_{2}\right) e_{2} \\
& =\mathcal{P}_{1}(\xi) e_{1}+\mathcal{P}_{2}(\xi) e_{2},
\end{aligned}
$$

where the projections $\mathcal{P}_{1}: \mathbb{C}_{2} \rightarrow \mathbb{C}$ and $\mathcal{P}_{2}: \mathbb{C}_{2} \rightarrow \mathbb{C}$ are defined as $\mathcal{P}_{1}\left(z_{1}+i_{2} z_{2}\right)=z_{1}-i_{1} z_{2}$ and $\mathcal{P}_{1}\left(z_{1}+i_{2} z_{2}\right)=$ $z_{1}+i_{1} z_{2}$, respectively. $\left\{e_{1}, e_{2}\right\}$ is idempotent basis of bicomplex numbers. For further details of bicomplex holomorphic functions and bicomplex numbers you can refer [7, 10, 14].

\section{Bicomplex Triple Laplace Transform}

Let $f(x, y, z)$ be a bicomplex-valued function of three variables $x, y, z>0$, which is piecewise continuous and has exponential order $K_{1}, K_{2}$, and $K_{3}$ w.r.t. $x, y$, and $z$, respectively.
The bicomplex Laplace transform (see, Kumar and Kumar [5]) w.r.t. $x$ is

$$
\begin{aligned}
L_{x}[f(x, y, z) ; \xi] & =\int_{0}^{\infty} e^{-\xi x} f(x, y, z) d x \\
& =\bar{f}(\xi, y, z), \quad \xi \in \Omega_{1} \subset \mathbb{C}_{2}
\end{aligned}
$$

where

$$
\begin{aligned}
\Omega_{1}= & \left\{\xi=\xi_{1} e_{1}+\xi_{2} e_{2} \in \mathbb{C}_{2}: \operatorname{Re}\left(\mathcal{P}_{1}: \xi\right)>K_{1}\right. \\
& \text { and } \left.\operatorname{Re}\left(\mathcal{P}_{2}: \xi\right)>K_{1}\right\}
\end{aligned}
$$

or

$$
\Omega_{1}=\left\{\xi \in \mathbb{C}_{2}: \operatorname{Re}(\xi)>K_{1}+\left|\operatorname{Im}_{j}(\xi)\right|\right\}
$$

where $\operatorname{Im}_{j}(\xi)$ denotes the imaginary part of $\xi$ w.r.t. $j$. The integral in (4) is convergent and bicomplex holomorphic in $\Omega_{1}$. Similarly, bicomplex Laplace transform of $f(x, y, z)$ w.r.t. $y$ is

$$
\begin{aligned}
L_{y}[f(x, y, z) ; \eta] & =\int_{0}^{\infty} e^{-\eta y} f(x, y, z) d y \\
& =\bar{f}(x, \eta, z), \quad \eta \in \Omega_{2} \subset \mathbb{C}_{2}
\end{aligned}
$$

where

$$
\begin{aligned}
\Omega_{2}= & \left\{\eta=\eta_{1} e_{1}+\eta_{2} e_{2} \in \mathbb{C}_{2}: \operatorname{Re}\left(\mathcal{P}_{1}: \eta\right)>K_{2}\right. \\
& \text { and } \left.\operatorname{Re}\left(\mathcal{P}_{2}: \eta\right)>K_{2}\right\}
\end{aligned}
$$

or

$$
\Omega_{2}=\left\{\eta \in \mathbb{C}_{2}: \operatorname{Re}(\eta)>K_{2}+\left|\operatorname{Im}_{j}(\eta)\right|\right\}
$$

where (7) is convergent and bicomplex holomorphic in $\Omega_{2}$. And bicomplex Laplace transform of $f(x, y, z)$ w.r.t. $z$ is

$$
\begin{aligned}
L_{z}[f(x, y, z) ; \gamma] & =\int_{0}^{\infty} e^{-\gamma z} f(x, y, z) d z \\
& =\bar{f}(x, y, \gamma), \quad \gamma \in \Omega_{3} \subset \mathbb{C}_{2}
\end{aligned}
$$

where

$$
\begin{aligned}
\Omega_{3}= & \left\{\gamma=\gamma_{1} e_{1}+\gamma_{2} e_{2} \in \mathbb{C}_{2}: \operatorname{Re}\left(\mathcal{P}_{1}: \eta\right)>K_{3}\right. \\
& \text { and } \left.\operatorname{Re}\left(\mathcal{P}_{2}: \eta\right)>K_{3}\right\}
\end{aligned}
$$

or

$$
\Omega_{3}=\left\{\gamma \in \mathbb{C}_{2}: \operatorname{Re}(\gamma)>K_{3}+\left|\operatorname{Im}_{j}(\gamma)\right|\right\}
$$

Now, taking the bicomplex Laplace transform of (4) w.r.t. $y$ and $z$ and using (7) and (10), we have

$$
\begin{aligned}
& L_{x, y, z}[f(x, y, z) ; \xi, \eta, \gamma] \\
& =\int_{0}^{\infty} \int_{0}^{\infty} \int_{0}^{\infty} e^{-(\xi x+\eta y+\gamma z)} f(x, y, z) d x d y d z \\
& =\overline{\bar{f}}(\xi, \eta, \gamma), \quad(\xi, \eta, \gamma) \in \Omega
\end{aligned}
$$

the integral on right hand side is convergent and bicomplex holomorphic in

$$
\Omega=\left\{(\xi, \eta, \gamma) \in \mathbb{C}_{2}^{3}: \xi \in \Omega_{1}, \eta \in \Omega_{2}, \text { and } \gamma \in \Omega_{3}\right\} .
$$

Now, we define the bicomplex triple Laplace transform as follows: 
Definition 3.1. Let $f(x, y, z)$ be a bicomplex-valued function of three variables $x, y, z>0$, which is piecewise continuous and has exponential order $K_{1}, K_{2}$, and $K_{3}$ w.r.t. $x, y$, and $z$, respectively. We call the transform in (13) bicomplex triple Laplace transform.

\section{Properties of Bicomplex Triple Laplace Transform}

In this section, we present some fundamental properties of bicomplex triple Laplace transform.

Proposition 4.1 (Linearity Property). Let $\overline{\bar{f}}(\xi, \eta, \gamma)$ and $\overline{\bar{g}}(\xi, \eta, \gamma)$ be bicomplex triple Laplace transforms of two bicomplex-valued functions $f(x, y, z)$ and $g(x, y, z)$ of $x, y, z>0$ with ROC $\Omega_{1}$ and $\Omega_{2}$, respectively. Then

$$
\begin{aligned}
& L_{x, y, z}\left[c_{1} f(x, y, z)+c_{2} g(x, y, z)\right] \\
& =c_{1} \overline{\bar{f}}(\xi, \eta, \gamma)+c_{2} \overline{\bar{g}}(\xi, \eta, \gamma), \quad(\xi, \eta, \gamma) \in \Omega=\Omega_{1} \cap \Omega_{2} .
\end{aligned}
$$

where $c_{1}$ and $c_{2}$ are arbitrary constants.

Proof. From the definition (13), we have

$$
\begin{aligned}
& L_{x, y, z}\left[c_{1} f(x, y, z)+c_{2} g(x, y, z)\right]= \\
& \left.\int_{0}^{\infty} \int_{0}^{\infty} \int_{0}^{\infty} e^{-(\xi x+\eta y+\gamma z}\right)\left[c_{1} f(x, y, z)+c_{2} g(x, y, z)\right] d x d y d z \\
= & c_{1} \int_{0}^{\infty} \int_{0}^{\infty} \int_{0}^{\infty} e^{-(\xi x+\eta y+\gamma z)} f(x, y, z) d x d y d z \\
& +c_{2} \int_{0}^{\infty} \int_{0}^{\infty} \int_{0}^{\infty} e^{-(\xi x+\eta y+\gamma z)} g(x, y, z) d x d y d z \\
= & c_{1} \overline{\bar{f}}(\xi, \eta, \gamma)+c_{2} \overline{\bar{g}}(\xi, \eta, \gamma) .
\end{aligned}
$$

Thus,

$$
\begin{aligned}
& L_{x, y, z}\left[c_{1} f(x, y, z)+c_{2} g(x, y, z)\right] \\
& =c_{1} \overline{\bar{f}}(\xi, \eta, \gamma)+c_{2} \overline{\bar{g}}(\xi, \eta, \gamma) .
\end{aligned}
$$

Proposition 4.2 (Change of Scale Property). Let $f(x, y, z)$ be bicomplex-valued function of $x, y, z>0$ such that $L_{x, y, z}[f(x, y, z)]=\overline{\bar{f}}(\xi, \eta, \gamma)$. Then

$$
L_{x, y, z}\left[f\left(\alpha_{1} x, \alpha_{2} y, \alpha_{3} z\right)\right]=\frac{1}{\alpha_{1} \alpha_{2} \alpha_{3}} \overline{\bar{f}}\left(\frac{\xi}{\alpha_{1}}, \frac{\eta}{\alpha_{2}}, \frac{\gamma}{\alpha_{3}}\right),
$$

$$
(\xi, \eta, \gamma) \in \Omega \text { and } \alpha_{1}, \alpha_{2}, \alpha_{3}>0
$$

Proof. From the definition (13), we have

$$
\begin{aligned}
& L_{x, y, z}\left[f\left(\alpha_{1} x, \alpha_{2} y, \alpha_{3} z\right)\right] \\
= & \int_{0}^{\infty} \int_{0}^{\infty} \int_{0}^{\infty} e^{-(\xi x+\eta y+\gamma z} f\left(\alpha_{1} x, \alpha_{2} y, \alpha_{3} z\right) d x d y d z \\
= & \int_{0}^{\infty} \int_{0}^{\infty} e^{-(\eta y+\gamma z)}\left(\int_{0}^{\infty} e^{-\xi x} f\left(\alpha_{1} x, \alpha_{2} y, \alpha_{3} z\right) d x\right) d y d z
\end{aligned}
$$$$
=\frac{1}{\alpha_{1}} \int_{0}^{\infty} \int_{0}^{\infty} e^{-(\eta y+\gamma z)}\left(\int_{0}^{\infty} e^{-\frac{\xi}{\alpha_{1}} p} f\left(p, \alpha_{2} y, \alpha_{3} z\right) d p\right) d y d z,
$$

[Taking $\alpha_{1} x=p$ ]

$=\frac{1}{\alpha_{1}} \int_{0}^{\infty} \int_{0}^{\infty} e^{-(\eta y+\gamma z)} \bar{f}\left(\frac{\xi}{\alpha_{1}}, \alpha_{2} y, \alpha_{3} z\right) d y d z$
$=\frac{1}{\alpha_{1} \alpha_{2}} \int_{0}^{\infty} e^{-\gamma z}\left(\int_{0}^{\infty} e^{-\frac{\eta}{\alpha_{2}} q} \bar{f}\left(\frac{\xi}{\alpha_{1}}, q, \alpha_{3} z\right) d q\right) d z$,

[Taking $\alpha_{2} y=q$ ]

$$
\begin{aligned}
& =\frac{1}{\alpha_{1} \alpha_{2}} \int_{0}^{\infty} e^{-\gamma z} \overline{\bar{f}}\left(\frac{\xi}{\alpha_{1}}, \frac{\eta}{\alpha_{2}}, \alpha_{3} z\right) d z \\
& =\frac{1}{\alpha_{1} \alpha_{2} \alpha_{3}} \int_{0}^{\infty} e^{-\frac{\gamma}{\alpha_{3}} r} \overline{\bar{f}}\left(\frac{\xi}{\alpha_{1}}, \frac{\eta}{\alpha_{2}}, r\right) d z,\left[\text { Taking } \alpha_{3} z=r\right] \\
& =\frac{1}{\alpha_{1} \alpha_{2} \alpha_{3}} \overline{\bar{f}}\left(\frac{\xi}{\alpha_{1}}, \frac{\eta}{\alpha_{2}}, \frac{\gamma}{\alpha_{3}}\right) .
\end{aligned}
$$

Thus,

$$
L_{x, y, z}\left[f\left(\alpha_{1} x, \alpha_{2} y, \alpha_{3} z\right)\right]=\frac{1}{\alpha_{1} \alpha_{2} \alpha_{3}} \overline{\bar{f}}\left(\frac{\xi}{\alpha_{1}}, \frac{\eta}{\alpha_{2}}, \frac{\gamma}{\alpha_{3}}\right) .
$$

Proposition 4.3 (First Shifting Property). Let $f(x, y, z)$ be bicomplex-valued function of $x, y, z>0$ such that $L_{x, y, z}[f(x, y, z)]=\overline{\bar{f}}(\xi, \eta, \gamma)$. Then

$$
\begin{array}{r}
L_{x, y, z}\left[e^{a x+b y+c z} f(x, y, z)\right]=\overline{\overline{\bar{f}}}(\xi-a, \eta-b, \gamma-c), \\
(\xi-a, \eta-b, \gamma-c) \in \Omega
\end{array}
$$

where $\Omega$ defined in (14).

Proof. From the definition (13), we have

$$
\begin{aligned}
& L_{x, y, z}\left[e^{a x+b y+c z} f(x, y, z)\right] \\
= & \int_{0}^{\infty} \int_{0}^{\infty} \int_{0}^{\infty} e^{-(\xi x+\eta y+\gamma z)} e^{a x+b y+c z} f(x, y, z) d x d y d z \\
= & \int_{0}^{\infty} \int_{0}^{\infty} e^{-(\eta-b) y-(\gamma-c) z}\left(\int_{0}^{\infty} e^{-(\xi-a) x} f(x, y, z) d x\right) d y d z \\
= & \int_{0}^{\infty} e^{-(\gamma-c) z}\left(\int_{0}^{\infty} e^{-(\eta-b) y} \bar{f}(\xi-a, y, z) d y\right) d z \\
= & \int_{0}^{\infty} e^{-(\gamma-c) z} \overline{\bar{f}}(\xi-a, \eta-b, z) d z \\
= & \overline{\bar{f}}(\xi-a, \eta-b, \gamma-c) .
\end{aligned}
$$

Thus,

$$
L_{x, y, z}\left[e^{a x+b y+c z} f(x, y, z)\right]=\overline{\bar{f}}(\xi-a, \eta-b, \gamma-c) .
$$


Proposition 4.4 (Triple Laplace Transform of Derivatives). Let $f(x, y, z)$ be bicomplex-valued function of $x, y, z>0$ such that $L_{x, y, z}[f(x, y, z)]=\overline{\bar{f}}(\xi, \eta, \gamma)$. Then

$$
\begin{aligned}
& L_{x, y, z}\left[f_{x y z}(x, y, z)\right] \\
& =\xi \eta \gamma \overline{\bar{f}}(\xi, \eta, \gamma)-\xi \eta \overline{\bar{f}}(\xi, \eta, 0)-\eta \gamma \overline{\bar{f}}(0, \eta, \gamma)-\gamma \xi \overline{\bar{f}}(\xi, 0, \gamma) \\
& \quad+\xi \bar{f}(\xi, 0,0)+\eta \bar{f}(0, \eta, 0)+\gamma \bar{f}(0,0, \gamma)-f(0,0,0),
\end{aligned}
$$$$
(\xi, \eta, \gamma) \in \Omega
$$

where $\Omega$ defined in (14) and

$f_{x y z}(x, y, z)=\frac{\partial^{3}}{\partial x \partial y \partial z} f(x, y, z)$.

Proof. From the definition (13), we have

$$
\begin{aligned}
& L_{x, y, z}\left[f_{x y z}(x, y, z)\right] \\
& =\int_{0}^{\infty} \int_{0}^{\infty} e^{-(\eta y+\gamma z)}\left[e^{-\xi x} f_{x y z}(x, y, z) d x\right] d y d z \\
& =\int_{0}^{\infty} \int_{0}^{\infty} e^{-(\eta y+\gamma z)}\left[\left.e^{-\xi x} f_{y z}(x, y, z)\right|_{x=0} ^{\infty}\right. \\
& \left.\quad+\xi \int_{0}^{\infty} e^{-\xi x} f_{y z}(x, y, z) d x\right] d y d z
\end{aligned}
$$

$=\int_{0}^{\infty} \int_{0}^{\infty} e^{-(\eta y+\gamma z)}\left[-f_{y z}(0, y, z)+\xi \bar{f}_{y z}(\xi, y, z)\right] d y d z$

$=\int_{0}^{\infty} e^{-\gamma z}\left[-\int_{0}^{\infty} e^{-\eta y} f_{y z}(0, y, z) d y\right.$

$\left.+\xi \int_{0}^{\infty} e^{-\eta y} \bar{f}_{y z}(\xi, y, z) d y\right] d z$

$=\int_{0}^{\infty} e^{-\gamma z}\left[-\left.e^{-\eta y} f_{z}(0, y, z)\right|_{y=0} ^{\infty}-\eta \int_{0}^{\infty} e^{-\eta y} f_{z}(0, y, z) d z\right.$

$\left.+\xi\left\{\left.e^{-\eta y} \bar{f}_{z}(\xi, y, z)\right|_{y=0} ^{\infty}+\eta \int_{0}^{\infty} e^{-\eta y} \bar{f}_{z}(\xi, y, z) d y\right\}\right] d z$

$=\int_{0}^{\infty} e^{-\gamma z}\left[f_{z}(0,0, z)-\eta \bar{f}_{z}(0, \eta, z)-\xi \bar{f}_{z}(\xi, 0, z)\right.$

$\left.+\xi \eta \overline{\bar{f}}_{z}(\xi, \eta, z)\right] d z$

$=\int_{0}^{\infty} e^{-\gamma z} f_{z}(0,0, z) d z-\eta \int_{0}^{\infty} e^{-\gamma z} \bar{f}_{z}(0, \eta, z) d z$

$-\xi \int_{0}^{\infty} e^{-\gamma z} \bar{f}_{z}(\xi, 0, z) d z+\xi \eta \int_{0}^{\infty} e^{-\gamma z} \overline{\bar{f}}_{z}(\xi, \eta, z) d z$

$=\left[\left.e^{-\gamma z} f(0,0, z)\right|_{0} ^{\infty}\right]+\gamma \int_{0}^{\infty} e^{-\gamma z} f(0,0, z) d z$

$-\eta\left[\left.e^{-\gamma z} \bar{f}(0, \eta, z)\right|_{0} ^{\infty}\right]-\eta \gamma \int_{0}^{\infty} e^{-\gamma z} \bar{f}(0, \eta, z) d z$

$-\xi\left[\left.e^{-\gamma z} \bar{f}(\xi, 0, z)\right|_{0} ^{\infty}\right]-\xi \gamma \int_{0}^{\infty} e^{-\gamma z} \bar{f}(\xi, 0, z) d z$

$+\xi \eta\left[\left.e^{-\gamma z} \overline{\bar{f}}(\xi, \eta, z)\right|_{0} ^{\infty}\right]+\xi \eta \gamma \int_{0}^{\infty} e^{-\gamma z} \overline{\bar{f}}(\xi, \eta, z) d z$

$=\xi \eta \gamma \overline{\overline{\bar{f}}}(\xi, \eta, \gamma)-\xi \eta \overline{\bar{f}}(\xi, \eta, 0)-\eta \gamma \overline{\bar{f}}(0, \eta, \gamma)-\gamma \xi \overline{\bar{f}}(\xi, 0, \gamma)$

$+\xi \bar{f}(\xi, 0,0)+\eta \bar{f}(0, \eta, 0)+\gamma \bar{f}(0,0, \gamma)-f(0,0,0)$.
Proposition 4.5. Let $f(x, y, z)$ be bicomplex-valued function of $x, y, z>0$ such that $L_{x, y, z}[f(x, y, z)]=\overline{\bar{f}}(\xi, \eta, \gamma)$. Then

$$
L_{x, y, z}[x y z f(x, y, z)]=-\frac{\partial^{3}}{\partial \xi \partial \eta \partial \gamma} \overline{\bar{f}}(\xi, \eta, \gamma),
$$

where $\Omega$ defined in (14).

Proof. From the definition (13), we have

$$
\begin{aligned}
& \frac{\partial^{3}}{\partial \xi \partial \eta \partial \gamma} \overline{\bar{f}}(\xi, \eta, \gamma) \\
= & \left(\frac{\partial^{3} \overline{\bar{f}}_{e_{1}}\left(\xi_{1}, \eta_{1}, \gamma_{1}\right)}{\partial \xi_{1} \partial \eta_{1} \partial \gamma_{1}}\right) e_{1}+\left(\frac{\partial^{3} \overline{\bar{f}}_{e_{2}}\left(\xi_{2}, \eta_{2}, \gamma_{2}\right)}{\partial \xi_{2} \partial \eta_{2} \partial \gamma_{2}}\right) e_{2} \\
= & \left(\frac{\partial^{3}}{\partial \xi_{1} \partial \eta_{1} \partial \gamma_{1}} \int_{0}^{\infty} \int_{0}^{\infty} \int_{0}^{\infty} e^{-\xi_{1} x-\eta_{1} y-\gamma_{1} z} f_{e_{1}}(x, y, z) d x d y d z\right) e_{1} \\
+ & \left(\frac{\partial^{3}}{\partial \xi_{2} \partial \eta_{2} \partial \gamma_{2}} \int_{0}^{\infty} \int_{0}^{\infty} \int_{0}^{\infty} e^{-\xi_{2} x-\eta_{2} y-\gamma_{2} z} f_{e_{2}}(x, y, z) d x d y d z\right) e_{2}
\end{aligned}
$$

where $\overline{\overline{\bar{f}}}(\xi, \eta, \gamma)=\overline{\overline{\bar{f}}}_{e_{1}}\left(\xi_{1}, \eta_{1}, \gamma_{1}\right) e_{1}+\overline{\overline{\bar{f}}}_{e_{2}}\left(\xi_{2}, \eta_{2}, \gamma_{2}\right) e_{2}$, $\xi=\xi_{1} e_{1}+\xi_{2} e_{2}, \eta=\eta_{1} e_{1}+\eta_{2} e_{2}$, and $\gamma=\gamma_{1} e_{1}+\gamma_{2} e_{2}$. Applying Leibniz's rule for complex functions [13, p. 243], we get

$$
\begin{aligned}
& \frac{\partial^{3}}{\partial \xi \partial \eta \partial \gamma} \overline{\bar{f}}(\xi, \eta, \gamma) \\
= & (-1)^{3}\left\{\left(\int_{0}^{\infty} \int_{0}^{\infty} \int_{0}^{\infty} e^{-\xi_{1} x-\eta_{1} y-\gamma_{1} z} x y z f_{e_{1}}(x, y, z) d x d y d z\right) e_{1}\right. \\
& \left.+\left(\int_{0}^{\infty} \int_{0}^{\infty} \int_{0}^{\infty} e^{-\xi_{2} x-\eta_{2} y-\gamma_{2} z} x y z f_{e_{2}}(x, y, z) d x d y d z\right) e_{2}\right\} \\
= & \int_{0}^{\infty} \int_{0}^{\infty} \int_{0}^{\infty} e^{-\left(\xi_{1} e_{1}+\xi_{2} e_{2}\right) x-\left(\eta_{1} e_{1}+\eta_{2} e_{2}\right) y-\left(\gamma_{1} e_{1}+\gamma_{2} e_{2}\right) z} x y z \\
& \times\left(f_{e_{1}}(x, y, z) e_{1}+f_{e_{2}}(x, y, z) e_{2}\right) d x d y d z \\
= & \int_{0}^{\infty} \int_{0}^{\infty} \int_{0}^{\infty} e^{-\xi x-\eta y-\gamma z} x y z f(x, y, z) d x d y d z .
\end{aligned}
$$

Thus,

$$
L_{x, y, z}[x y z f(x, y, z)]=-\frac{\partial^{3}}{\partial \xi \partial \eta \partial \gamma} \overline{\bar{f}}(\xi, \eta, \gamma) .
$$

In general

$$
\begin{aligned}
& L_{x, y, z}\left[x^{l} y^{m} z^{n} f(x, y, z)\right] \\
= & (-1)^{l+m+n} \frac{\partial^{l+m+n}}{\partial \xi^{l} \partial \eta^{m} \partial \gamma^{n}} \overline{\bar{f}}(\xi, \eta, \gamma) .
\end{aligned}
$$

Proposition 4.6 (Division by $x y z)$. Let $f(x, y, z)$ be bicomplex-valued function of $x, y, z>0$ such that $L_{x, y, z}[f(x, y, z)]=\overline{\bar{f}}(\xi, \eta, \gamma)$. Then

$$
L_{x, y, z}\left[\frac{f(x, y, z)}{x y z}\right]=\int_{\xi}^{\infty} \int_{\eta}^{\infty} \int_{\gamma}^{\infty} \overline{\bar{f}}(\xi, \eta, \gamma) d \xi d \eta d \gamma
$$$$
(\xi, \eta, \gamma) \in \Omega
$$

where $\Omega$ defined in (14) and provided the integral on right hand exists. 
Proof. From the definition (13), we have

$\overline{\bar{f}}(\xi, \eta, \gamma)=\int_{0}^{\infty} \int_{0}^{\infty} \int_{0}^{\infty} e^{-\xi x-\eta y-\gamma z} f(x, y, z) d x d y d z$

$$
\begin{aligned}
& \overline{\bar{f}}(\xi, \eta, \gamma)=\overline{\overline{\bar{f}}}_{e_{1}}\left(\xi_{1}, \eta_{1}, \gamma_{1}\right) e_{1}+\overline{\bar{f}}_{e_{2}}\left(\xi_{2}, \eta_{2}, \gamma_{2}\right) e_{2} \\
= & \left(\int_{0}^{\infty} \int_{0}^{\infty} \int_{0}^{\infty} e^{-\xi_{1} x-\eta_{1} y-\gamma_{1} z} f_{e_{1}}(x, y, z) d x d y d z\right) e_{1} \\
& +\left(\int_{0}^{\infty} \int_{0}^{\infty} \int_{0}^{\infty} e^{-\xi_{2} x-\eta_{2} y-\gamma_{2} z} f_{e_{2}}(x, y, z) d x d y d z\right) e_{2} .
\end{aligned}
$$

Integrating (16) w.r.t. $\xi, \eta$, and $\gamma$ from $\xi$ to $\infty, \eta$ to $\infty$, and $\gamma$ to $\infty$, respectively, we have

$$
\begin{aligned}
& \int_{\xi}^{\infty} \int_{\eta}^{\infty} \int_{\gamma}^{\infty} \overline{\bar{f}}(\xi, \eta, \gamma) d \xi d \eta d \gamma \\
= & \left(\int_{\xi_{1}}^{\infty} \int_{\eta_{1}}^{\infty} \int_{\gamma_{1}}^{\infty} \overline{\bar{f}}_{e_{1}}\left(\xi_{1}, \eta_{1}, \gamma_{1}\right) d \xi_{1} d \eta_{1} d \gamma_{1}\right) e_{1} \\
& +\left(\int_{\xi_{2}}^{\infty} \int_{\eta_{2}}^{\infty} \int_{\gamma_{2}}^{\infty} \overline{\bar{f}}_{e_{2}}\left(\xi_{2}, \eta_{2}, \gamma_{2}\right) d \xi_{2} d \eta_{2} d \gamma_{2}\right) e_{2} \\
= & {\left[\int _ { \xi _ { 1 } } ^ { \infty } \int _ { \eta _ { 1 } } ^ { \infty } \int _ { \gamma _ { 1 } } ^ { \infty } \left(\int_{0}^{\infty} \int_{0}^{\infty}\right.\right.} \\
& \left.\left.\times \int_{0}^{\infty} e^{-\xi_{1} x-\eta_{1} y-\gamma_{1} z} f_{e_{1}}(x, y, z) d x d y d z\right) d \xi_{1} d \eta_{1} d \gamma_{1}\right] e_{1} \\
& +\left[\int _ { \xi _ { 2 } } ^ { \infty } \int _ { \eta _ { 2 } } ^ { \infty } \int _ { \gamma _ { 2 } } ^ { \infty } \left(\int_{0}^{\infty} \int_{0}^{\infty}\right.\right. \\
& \left.\left.\times \int_{0}^{\infty} e^{-\xi_{2} x-\eta_{2} y-\gamma_{2} z} f_{e_{2}}(x, y, z) d x d y d z\right) d \xi_{2} d \eta_{2} d \gamma_{2}\right] e_{2} .
\end{aligned}
$$

By change of order of integration, we have

$$
\begin{array}{rl} 
& \int_{\xi}^{\infty} \int_{\eta}^{\infty} \int_{\gamma}^{\infty} \overline{\bar{f}}(\xi, \eta, \gamma) d \xi d \eta d \gamma \\
= & {\left[\int _ { 0 } ^ { \infty } \int _ { 0 } ^ { \infty } \int _ { 0 } ^ { \infty } \left(\int_{\xi_{1}}^{\infty} \int_{\eta_{1}}^{\infty}\right.\right.} \\
& \left.\left.\times \int_{\gamma_{1}}^{\infty} e^{-\xi_{1} x-\eta_{1} y-\gamma_{1} z} f_{e_{1}}(x, y, z) d \xi_{1} d \eta_{1} d \gamma_{1}\right) d x d y d z\right] e_{1} \\
& +\int_{0}^{\infty} \int_{0}^{\infty} \int_{0}^{\infty}\left(\int_{\xi_{2}}^{\infty} \int_{\eta_{2}}^{\infty}\right. \\
& \left.\left.\times \int_{\gamma_{2}}^{\infty} e^{-\xi_{2} x-\eta_{2} y-\gamma_{2} z} f_{e_{2}}(x, y, z) d \xi_{2} d \eta_{2} d \gamma_{2}\right) d x d y d z\right] e_{2} \\
= & \left(\int_{0}^{\infty} \int_{0}^{\infty} \int_{0}^{\infty} \frac{e^{-\xi_{1} x-\eta_{1} y-\gamma_{1} z}}{x y z} f_{e_{1}}(x, y, z) d x d y d z\right) e_{1} \\
& +\left(\int_{0}^{\infty} \int_{0}^{\infty} \int_{0}^{\infty} \frac{e^{-\xi_{2} x-\eta_{2} y-\gamma_{2} z}}{x y z} f_{e_{2}}(x, y, z) d x d y d z\right) e_{2} \\
= & \int_{0}^{\infty} \int_{0}^{\infty} \int_{0}^{\infty} e^{-\left(\xi_{1} e_{1}+\xi_{2} e_{2}\right) x-\left(\eta_{1} e_{1}+\eta_{2} e_{2}\right) y-\left(\gamma_{1} e_{1}+\gamma_{2} e_{2}\right) z} \\
& \times\left(\frac{f_{e_{1}}(x, y, z) e_{1}+f_{e_{2}}(x, y, z) e_{2}}{x y z}\right) d x d y d z \\
= & \int_{0}^{\infty} \int_{0}^{\infty} \int_{0}^{\infty} e^{-\xi x-\eta y-\gamma z} \frac{f(x, y, z)}{x y z} d x d y d z \\
& {[x, y, z)} \\
x y z & f
\end{array}
$$

Thus,

$$
L_{x, y, z}\left[\frac{f(x, y, z)}{x y z}\right]=\int_{\xi}^{\infty} \int_{\eta}^{\infty} \int_{\gamma}^{\infty} \overline{\bar{f}}(\xi, \eta, \gamma) d \xi d \eta d \gamma .
$$

Proposition 4.7 (Triple Laplace Transform of Integrals). Let $f(x, y, z)$ be bicomplex-valued function of $x, y, z>0$ such that $L_{x, y, z}[f(x, y, z)]=\overline{\bar{f}}(\xi, \eta, \gamma)$. Then

$$
\begin{aligned}
& L_{x, y, z}\left[\int_{0}^{x} \int_{0}^{y} \int_{0}^{z} f(u, v, w) d u d v d w\right]=\frac{\overline{\bar{f}}(\xi, \eta, \gamma)}{\xi \eta \gamma}, \\
& \operatorname{Re}(\xi)>\left|\operatorname{Im}_{j}(\xi)\right|, \operatorname{Re}(\eta)>\left|\operatorname{Im}_{j}(\eta)\right|, \operatorname{Re}(\gamma)>\left|\operatorname{Im}_{j}(\gamma)\right| .
\end{aligned}
$$

Proof. Let

$$
g(x, y, z)=\int_{0}^{x} \int_{0}^{y} \int_{0}^{z} f(u, v, w) d u d v d w .
$$

Hence, we have

$$
\begin{gathered}
g_{x y z}(x, y, z)=f(x, y, z) \text { and } g(0,0,0)=0 \\
\therefore \quad L_{x, y, z}\left[g_{x y z}(x, y, z)\right]=L[f(x, y, z)]=\overline{\bar{f}}(\xi, \eta, \gamma) .
\end{gathered}
$$

Now, from the Proposition 4.4, we have

$$
\begin{aligned}
& L_{x, y, z}\left[g_{x y z}(x, y, z)\right]=\xi \eta \gamma \overline{\bar{g}}(\xi, \eta, \gamma)-\xi \eta \overline{\bar{g}}(\xi, \eta, 0) \\
& -\eta \gamma \overline{\bar{g}}(0, \eta, \gamma)-\gamma \xi \overline{\bar{g}}(\xi, 0, \gamma)+\xi \bar{g}(\xi, 0,0)+\eta \bar{g}(0, \eta, 0) \\
& +\gamma \bar{g}(0,0, \gamma)-g(0,0,0) \\
& \quad \overline{\bar{f}}(\xi, \eta, \gamma)=\xi \eta \gamma \overline{\bar{g}}(\xi, \eta, \gamma)-\xi \eta \overline{\bar{g}}(\xi, \eta, 0)-\eta \gamma \overline{\bar{g}}(0, \eta, \gamma) \\
& -\gamma \xi \overline{\bar{g}}(\xi, 0, \gamma)+\xi \bar{g}(\xi, 0,0)+\eta \bar{g}(0, \eta, 0)+\gamma \bar{g}(0,0, \gamma)
\end{aligned}
$$


Therefore,

$$
\begin{aligned}
\overline{\bar{g}}(\xi, \eta, \gamma)= & \frac{\overline{\bar{f}}(\xi, \eta, \gamma)}{\xi \eta \gamma}+\frac{\overline{\bar{g}}(\xi, \eta, 0)}{\gamma}+\frac{\overline{\bar{g}}(0, \eta, \gamma)}{\xi}+\frac{\overline{\bar{g}}(\xi, 0, \gamma)}{\eta} \\
& -\frac{\bar{g}(\xi, 0,0)}{\eta \gamma}-\frac{\bar{g}(0, \eta, 0)}{\xi \gamma}-\frac{\bar{g}(0,0, \gamma)}{\xi \eta} .
\end{aligned}
$$

But $\overline{\bar{g}}(\xi, \eta, 0)=0, \overline{\bar{g}}(0, \eta, \gamma)=0, \overline{\bar{g}}(\xi, 0, \gamma)=0, \bar{g}(\xi, 0,0)=$ $0, \bar{g}(0, \eta, 0)=0$, and $\bar{g}(0,0, \gamma)=0$. Therefore,

$$
\begin{aligned}
& \overline{\bar{g}}(\xi, \eta, \gamma)=\frac{\overline{\bar{f}}(\xi, \eta, \gamma)}{\xi \eta \gamma} \\
\therefore \quad & L_{x, y, z}[g(x, y, z)]=\frac{\overline{\bar{f}}(\xi, \eta, \gamma)}{\xi \eta \gamma} .
\end{aligned}
$$

Hence,

$$
L_{x, y, z}\left[\int_{0}^{x} \int_{0}^{y} \int_{0}^{z} f(u, v, w) d u d v d w\right]=\frac{\overline{\bar{f}}(\xi, \eta, \gamma)}{\xi \eta \gamma} .
$$

Proposition 4.8. Let $f(x, y, z)$ be a periodic function of pe$\operatorname{riod} T_{1}, T_{2}$, and $T_{3}$ w.r.t. $x, y$, and $z$ respectively. Then the bicomplex triple Laplace transform is given by

$L_{x, y, z}[f(x, y, z)]=\frac{\int_{0}^{T_{1}} \int_{0}^{T_{2}} \int_{0}^{T_{3}} e^{-\xi x-\eta y-\gamma z} f(x, y, z) d x d y d z}{\left(1-e^{-T_{1} \xi}\right)\left(1-e^{-T_{2} \eta}\right)\left(1-e^{-T_{3} \gamma}\right)}$,

$$
\operatorname{Re}(\xi)>\left|\operatorname{Im}_{j}(\xi)\right|, \operatorname{Re}(\eta)>\left|\operatorname{Im}_{j}(\eta)\right| \text {, and } \operatorname{Re}(\gamma)>\left|\operatorname{Im}_{j}(\gamma)\right| .
$$

Proof. Let $f(x, y, z)$ be a periodic function with period $T_{1}$ w.r.t. $x$. Then for $\xi \in \mathbb{C}_{2}$ and $\operatorname{Re}(\xi)>\left|\operatorname{Im}_{j}(\xi)\right|$, see Agarwal et al. [21]

$$
L_{x}[f(x, y, z)]=\frac{\int_{0}^{T_{1}} e^{-\xi x} f(x, y, z) d x}{1-e^{T_{1} \xi}}=\bar{f}(\xi, y, z)
$$

Similarly, for $\eta \in \mathbb{C}_{2}$ and $\operatorname{Re}(\eta)>\left|\operatorname{Im}_{j}(\eta)\right|$ taking the bicomplex Laplace transform of (17) w.r.t. $y$, we have

$$
\begin{aligned}
& L_{y}[\bar{f}(\xi, y, z)]=\overline{\bar{f}}(\xi, y, z)=\frac{\int_{0}^{T_{2}} e^{-\eta y} \bar{f}(\xi, y, z) d y}{1-e^{-T_{2} \eta}} \\
= & \frac{1}{1-e^{-T_{2} \eta}} \int_{0}^{T_{2}} e^{-\eta y} \frac{\int_{0}^{T_{1}} e^{-\xi x} f(x, y, z) d x}{1-e^{T_{1} \xi}} d y \\
= & \frac{\int_{0}^{T_{1}} \int_{0}^{T_{2}} e^{-\xi x-\eta y} f(x, y, z) d x d y}{\left(1-e^{T_{1} \xi}\right)\left(1-e^{T_{2} \eta}\right)} .
\end{aligned}
$$

And for $\gamma \in \mathbb{C}_{2}$ and $\operatorname{Re}(\gamma)>\left|\operatorname{Im}_{j}(\gamma)\right|$ taking the bicomplex Laplace transform of (18) w.r.t. $z$, we have

$$
\begin{aligned}
& L_{z}[\overline{\bar{f}}(\xi, \eta, z)]=\overline{\bar{f}}(\xi, \eta, \gamma)=\frac{\int_{0}^{T_{3}} e^{-\gamma z} \overline{\bar{f}}(\xi, \eta, z) d z}{1-e^{-T_{3} \gamma}} \\
= & \frac{1}{1-e^{-T_{3} \gamma}} \int_{0}^{T_{3}} e^{-\gamma z} \frac{\int_{0}^{T_{1}} \int_{0}^{T_{2}} e^{-\xi x-\eta y} f(x, y, z) d x d y}{\left(1-e^{T_{1} \xi}\right)\left(1-e^{T_{2} \eta}\right)} d z \\
= & \frac{\int_{0}^{T_{1}} \int_{0}^{T_{2}} \int_{0}^{T_{3}} e^{-\xi x-\eta y-\gamma z} f(x, y, z) d x d y d z}{\left(1-e^{-T_{1} \xi}\right)\left(1-e^{-T_{2} \eta}\right)\left(1-e^{-T_{3} \gamma}\right)} .
\end{aligned}
$$

Thus,

$L_{x, y, z}[f(x, y, z)]=\frac{\int_{0}^{T_{1}} \int_{0}^{T_{2}} \int_{0}^{T_{3}} e^{-\xi x-\eta y-\gamma z} f(x, y, z) d x d y d z}{\left(1-e^{-T_{1} \xi}\right)\left(1-e^{-T_{2} \eta}\right)\left(1-e^{-T_{3} \gamma}\right)}$.

\section{Inversion}

In this section, we derive the inversion formula for bicomplex triple Laplace transform.

Proposition 5.1. Let $f(x, y, z)$ be bicomplex-valued function of $x, y, z>0$ such that $L_{x, y, z}[f(x, y, z)]=\overline{\overline{\bar{f}}}(\xi, \eta, \gamma)$. Then

$f(x, y, z)=-\frac{1}{8 \pi^{3} i_{1}} \int_{\Gamma_{1}} \int_{\Gamma_{2}} \int_{\Gamma_{3}} e^{\xi x+\eta y+\gamma z} \overline{\bar{f}}(\xi, \eta, \gamma) d \xi d \eta d \gamma$, $(\xi, \eta, \gamma) \in \Omega$

where $\Omega$ defined in (14) and $\Gamma_{1}, \Gamma_{2}$, and $\Gamma_{3}$ are Bromwich closed contours in bicomplex space.

Proof. Taking the inverse bicomplex Laplace transform [3] of $\overline{\bar{f}}(\xi, \eta, \gamma)$ w.r.t. $\gamma$, we get

$$
L_{\gamma}^{-1}[\overline{\bar{f}}(\xi, \eta, \gamma)]=\overline{\bar{f}}(\xi, \eta, z)=\frac{1}{2 \pi i_{1}} \int_{\Gamma_{3}} e^{\gamma z} \overline{\bar{f}}(\xi, \eta, \gamma) d \gamma
$$

Similarly, taking inverse bicomplex Laplace transform of (19) w.r.t. $\eta$, we have

$$
\begin{aligned}
L_{\eta}^{-1}[\overline{\bar{f}}(\xi, \eta, z)] & =\bar{f}(\xi, y, z)=\frac{1}{2 \pi i_{1}} \int_{\Gamma_{2}} e^{\eta y} \overline{\bar{f}}(\xi, \eta, z) d \eta \\
& =\frac{1}{\left(2 \pi i_{1}\right)^{2}} \int_{\Gamma_{2}} e^{\eta y}\left(\int_{\Gamma_{3}} e^{\gamma z} \overline{\bar{f}}(\xi, \eta, \gamma) d \gamma\right) d \eta \\
& =-\frac{1}{4 \pi^{2}} \int_{\Gamma_{2}} \int_{\Gamma_{3}} e^{\eta y+\gamma z} \overline{\bar{f}}(\xi, \eta, \gamma) d \eta d \gamma
\end{aligned}
$$

And, taking inverse bicomplex Laplace transform of (20) w.r.t. $\xi$, we have

$$
\begin{aligned}
& L_{\xi}^{-1}[\bar{f}(\xi, y, z)]=f(x, y, z)=\frac{1}{2 \pi i_{1}} \int_{\Gamma_{1}} e^{\xi x} \bar{f}(\xi, y, z) d \xi \\
= & -\frac{1}{8 \pi^{3} i_{1}} \int_{\Gamma_{1}} e^{\xi x}\left(\int_{\Gamma_{2}} \int_{\Gamma_{3}} e^{\eta y+\gamma z} \overline{\bar{f}}(\xi, \eta, \gamma) d \eta d \gamma\right) d \xi \\
= & -\frac{1}{8 \pi^{3} i_{1}} \int_{\Gamma_{1}} \int_{\Gamma_{2}} \int_{\Gamma_{3}} e^{\xi x+\eta y+\gamma z} \overline{\bar{f}}(\xi, \eta, \gamma) d \xi d \eta d \gamma .
\end{aligned}
$$

Hence,

$f(x, y, z)=-\frac{1}{8 \pi^{3} i_{1}} \int_{\Gamma_{1}} \int_{\Gamma_{2}} \int_{\Gamma_{3}} e^{\xi x+\eta y+\gamma z} \overline{\bar{f}}(\xi, \eta, \gamma) d \xi d \eta d \gamma$.

\section{Application}

In this section, we present application of bicomplex triple Laplace transform in finding the solution of some kind of thirdorder partial differential equation of bicomplex-valued function.

Consider the third order partial differential equation of the type

$$
\frac{\partial^{3} u(x, y, z)}{\partial x \partial y \partial z}+u(x, y, z)=f(x, y, z), \quad x, y, z>0
$$


where $u: \mathbb{R}^{3} \rightarrow \mathbb{C}_{2}$ and $f: \mathbb{R}^{3} \rightarrow \mathbb{C}_{2}$ can be expressed explicitly as

$u(x, y, z)=u_{0}(x, y, z)+i_{1} u_{1}(x, y, z)+i_{2} u_{2}(x, y, z)+$ $i_{1} i_{2} u_{3}(x, y, z)$ and $f(x, y, z)=f_{0}(x, y, z)+i_{1} f_{1}(x, y, z)+$ $i_{2} f_{2}(x, y, z)+i_{1} i_{2} f_{3}(x, y, z)$.

Taking bicomplex triple Laplace transform of (21) on both sides and applying Proposition 4.4, we have

$$
\begin{aligned}
& \xi \eta \gamma \overline{\bar{u}}(\xi, \eta, \gamma)-\xi \eta \overline{\bar{u}}(\xi, \eta, 0)-\eta \gamma \overline{\bar{u}}(0, \eta, \gamma)-\gamma \xi \overline{\bar{u}}(\xi, 0, \gamma) \\
& +\xi \bar{u}(\xi, 0,0)+\eta \bar{u}(0, \eta, 0)+\gamma \bar{u}(0,0, \gamma)-u(0,0,0) \\
& +\overline{\bar{u}}(\xi, \eta, \gamma)=\overline{\bar{f}}(\xi, \eta, \gamma) \\
& \Rightarrow \overline{\bar{u}}(\xi, \eta, \gamma)=\frac{\overline{\bar{f}}(\xi, \eta, \gamma)}{1+\xi \eta \gamma}+\frac{\xi \eta}{1+\xi \eta \gamma} \overline{\bar{u}}(\xi, \eta, 0)+ \\
& \quad \frac{\eta \gamma}{1+\xi \eta \gamma} \overline{\bar{u}}(0, \eta, \gamma)+\frac{\gamma \xi}{1+\xi \eta \gamma} \overline{\bar{u}}(\xi, 0, \gamma)-\frac{\xi}{1+\xi \eta \gamma} \bar{u}(\xi, 0,0) \\
& -\frac{\eta}{1+\xi \eta \gamma} \bar{u}(0, \eta, 0)-\frac{\gamma}{1+\xi \eta \gamma} \bar{u}(0,0, \gamma)+\frac{u(0,0,0)}{1+\xi \eta \gamma}
\end{aligned}
$$

Applying the inversion formula for bicomplex triple Laplace transform, we have

$$
\begin{gathered}
u(x, y, z)=-\frac{1}{8 \pi^{3} i_{1}} \int_{\Gamma_{1}} \int_{\Gamma_{2}} \int_{\Gamma_{3}} e^{\xi x+\eta y+\gamma z} \overline{\bar{u}}(\xi, \eta, \gamma) d \xi d \eta d \gamma \\
(\xi, \eta, \gamma) \in \Omega
\end{gathered}
$$

where $\Omega$ defined in (14), $\overline{\bar{u}}(\xi, \eta, \gamma)$ defined in (22), and $\Gamma_{1}, \Gamma_{2}$, and $\Gamma_{3}$ are Bromwich closed contours in bicomplex space.

\section{Conclusion}

In this article, we derive bicomplex triple Laplace transform and its properties which is a natural extension of the complex triple Laplace transform [1]. It is applicable in solving some kind of third-order partial differential equation of bicomplexvalued function due to large class of frequency domain. Bicomplex numbers being basically four dimensional hypercomplex numbers, provide large class of frequency domain.

\section{Acknowledgements}

The authors are very grateful to experts for their appropriate and constructive suggestions to improve this article.

\section{REFERENCES}

[1] A. Atangana, A note on the triple Laplace transform and its applications to some kind of third-order differential equation, Abstract and Applied Analysis, 2013, Article id 769102, 1-10, 2013.
[2] A. Banerjee, On the quantum mechanics of bicomplex Hamiltonian system, Annals of Physics, 377, 493-505, 2017.

[3] A. Banerjee, S.K. Datta, and A. Hoque, Inverse Laplace transform for Bi-complex variables, Mathematical inverse problems, 1(1), 8-14, 2014.

[4] A. Ghanmi and K. Zine, Bicomplex analogs of SegalBargmann and fractional Fourier transforms, Adv. Appl. Clifford Algebras, 29(4), Paper No. 74, 1-20, 2019.

[5] A. Kumar and P. Kumar, Bicomplex version of Laplace Transform, International Journal of Engg. and Tech., 3(3), 225-232, 2011.

[6] C. Segre, Le rappresentazioni reale delle forme complesse'e Gli Enti Iperalgebrici, Math. Ann., 40, 413-467, 1892.

[7] D. Alpay, M.E. Luna-Elizarrarás, M. Shapiro, and D.C. Struppa, Basics of Functional Analysis with Bicomplex Scalars, and Bicomplex Schur Analysis, Springer Briefs in Mathematics, Springer, Cham, 2014.

[8] D. Rochon and S. Tremblay, Bicomplex Quantum Mechanics: I. The generalized Schrödinger equation, Advances in Applied Clifford Algebras, 14(2), 231-248, 2004.

[9] D. Rochon and S. Tremblay, Bicomplex Quantum Mechanics: II. the Hilbert space, Advances in Applied Clifford Algebras, 16, 135-157, 2006.

[10] G.B. Price, An Introduction to Multicomplex Spaces and Functions, Marcel Dekker Inc., New York, 1991.

[11] G.S. Dragoni, Sulle funzioni olomorfe di una variable bicomplessa, Reale Acad. d'Italia Mem. Class Sci. Fic. Mat. Nat., 5, 597-665, 1934.

[12] J.C. Jaeger, The solution of boundary value problems by a double Laplace transformation, Bull. Am. Mathematical Soc., 46(8), 687-693, 1940.

[13] J.H. Mathew and R.W. Howell, Complex Analysis for Mathematics \& Engineering, Jones \& Bartlett Publishers, 2012.

[14] M.E. Luna-Elizarrarás, M. Shapiro, D.C. Struppa, and A. Vijiac, Bicomplex Holomorphic Functions : The Algebra, Geometry and Analysis of Bicomplex Numbers, Birkhäuser, ISBN 978-3-319-24866-4, 2015.

[15] M. Futagawa, On the theory of functions of quaternary variable-I, Tohoku Math. J., 29, 175-222, 1928.

[16] M. Futagawa, On the theory of functions of quaternary variable-II, Tohoku Math. J., 35, 69-120, 1932.

[17] P. cerejeiras, Yingxiong Fu, and N. Gomes, Bicomplex signals with sparsity constraints, Math. Methods Appl. Sci., 41(13), 5140-5148, 2018. 
[18] P.M. Humbert, Le Calcul Symbolique a Deux Variables, Annales de la Societe Scientifique, series A, 26, 1936.

[19] R. Agarwal, M.P. Goswami, and R.P. Agarwal, Bicomplex Version of Stieltjes Transform and Applications, $D y$ namics of Continuous, Discrete and Impulsive Systems Series B: Applications \& Algorithms, 21(4-5), 229-246, 2014.

[20] R. Agarwal, M.P. Goswami, and R.P. Agarwal, Bochner Theorem and Applications of Bicomplex Fourier-Stieltjes Transform, Advanced studies in contemporary Mathematics, 26(2), 355-369, 2016.

[21] R. Agarwal, M.P. Goswami, and R.P. Agarwal, Convolution Theorem and Applications of Bicomplex Laplace Transform, Advances in Mathematical Sciences and Applications, 24(1), 113-127, 2014.

[22] R. Agarwal, M.P. Goswami, and R.P. Agarwal, Double Laplace transform in bicomplex space with applications, Advances in Mathematical Sciences and Applications, 28(2), 255-271, 2019.

[23] R. Agarwal, M.P. Goswami, and R.P. Agarwal, Tauberian Theorem and Applications of Bicomplex LaplaceStieltjes Transform, Dynamics of Continuous, Discrete and Impulsive Systems, Series B: Applications \& Algorithms, 22, 141-153, 2015.
[24] R. Kumar and H. Saini, Topological bicomplex modules, Advances in Applied Clifford Algebra, 26, 1249-1270, 2016.

[25] R. Kumar, R. Kumar, and D. Rochon, The fundamental theorems in the framework of bicomplex topological modules, arXiv:1109.3424, 1-14, 2011.

[26] R.G. Lavoie, L. Marchildon, and D. Rochon, Finitedimensional bicomplex Hilbert space, Advances in Applied Clifford Algebra, 21, 561-581, 2011.

[27] R.G. Lavoie, L. Marchildon, and D. Rochon, Infinitedimensional bicomplex Hilbert space, Annals of Functional Analysis, 1(2), 75-91, 2010.

[28] R.G. Lavoie, L. Marchildon, and D. Rochon, The bicomplex quantum harmonic oscillator, IL Nuovo Cimento B, 125(10), 1173-1192, 2010.

[29] S. Rönn, Bicomplex algebra and function theory, arXiv:math/0101200v1 [math.CV], 1-71, 2001.

[30] T.A. Estrin and T.J. Higgins, The solution of boundary value problems by multiple Laplace transformations, Journal of Franklin Institute, 252(2), 153-167, 1951.

[31] Van Der Pol and K.F. Niessen, Simultaneous operational calculus, Philosophical Mag., 11, 368, 1936. 